\title{
3 Research Square

\section{Aberrant Functional and Effective Connectivity of the Frontostriatal Network in Unilateral Acute Tinnitus Patients with Hearing Loss}

\section{Gang-Ping Zhou}

The Affiliated Jiangning Hospital of Naning Medical University

\section{Yu-Chen Chen}

Nanjing First Hospital

\section{Wang-Wei Li}

The Affiliated Jiangning Hospital of Nanjing Medical University

\section{Heng-Le Wei}

The Affiliated Jiangning Hospital of Nanjing Medical University

\section{Yu-Sheng Yu}

The Affiliated Jiangning Hospital of Nanjing Medical University

\section{Qing-Qing Zhou}

The Affiliated Jiangning Hospital of Nanjing Medical University

\section{Xindao Yin}

Nanjing First Hospital

\section{Yue-Jin Tao}

The Affiliated Jiangning Hospital of Nanjing Medical University Hong Zhang ( $\square$ jnyyfsk@126.com)

The Affiliated Jiangning Hospital of Nanjing Medical University https://orcid.org/0000-0001-58272488

\section{Research Article}

Keywords: Functional connectivity, Frontostriatal Network, Granger causality analysis, Resting-state fMRI, Tinnitus

Posted Date: March 9th, 2021

DOl: https://doi.org/10.21203/rs.3.rs-202886/v1

License: (c) (1) This work is licensed under a Creative Commons Attribution 4.0 International License. Read Full License 
Version of Record: A version of this preprint was published at Brain Imaging and Behavior on July 23rd, 2021. See the published version at https://doi.org/10.1007/s11682-021-00486-9. 


\section{Aberrant Functional and Effective Connectivity of the Frontostriatal Network in Unilateral Acute Tinnitus Patients with Hearing Loss}

Gang-Ping Zhou ${ }^{1 \#}$, Yu-Chen Chen ${ }^{2 \#}$, Wang-Wei Li ${ }^{3}$, Heng-Le Wei ${ }^{1}$, Yu-Sheng Yu ${ }^{1}$, Qing-Qing Zhou ${ }^{1}$, Xindao Yin ${ }^{2}$, Yue-Jin Tao ${ }^{3 *}$ and Hong Zhang ${ }^{1 *}$

${ }^{1}$ Department of Radiology, The Affiliated Jiangning Hospital of Nanjing Medical University, No. 168, Gushan Road, Nanjing, Jiangsu Province, 211100, China;

${ }^{2}$ Department of Radiology, Nanjing First Hospital, Nanjing Medical University, Jiangsu Province, Nanjing, 210006, China;

${ }^{3}$ Department of E.N.T., The Affiliated Jiangning Hospital of Nanjing Medical University, No. 168, Gushan Road, Nanjing, Jiangsu Province, 211100, China.

*Correspondence:

Hong Zhang, Department of Radiology, The Affiliated Jiangning Hospital of Nanjing Medical University, No. 168, Gushan Road, Nanjing, Jiangsu Province, 211100, China. Email jnyyfsk@126.com. Tel: +86-25-51191100. Fax: +86-25-52281848

Yue-Jin Tao, Department of E.N.T., The Affiliated Jiangning Hospital of Nanjing Medical University, No. 168, Gushan Road, Nanjing, Jiangsu Province, 211100, China. Email1125266270@qq.com Tel: +86-25-52286929. Fax: +86-25-52281848

\#These authors contribute equally to this work 


\begin{abstract}
Purpose: The present study combined resting-state functional connectivity (FC) and Granger causality analysis (GCA) to explore frontostriatal network dysfunction in unilateral acute tinnitus (AT) patients with hearing loss.

Methods: The participants included 42 AT patients and 43 healthy control (HC) subjects who underwent resting-state functional magnetic resonance imaging (fMRI) scans. Based on the seed regions in the frontostriatal network, FC and GCA were conducted between the AT patients and HC subjects. Correlation analyses were used to examine correlations among altered FC values, GCA values, and clinical features in AT patients.
\end{abstract}

Results: Compared with HCs, AT patients showed a general reduction in $\mathrm{FC}$ between the seed regions in the frontostriatal network and nonauditory areas, including the frontal cortices, midcingulate cortex (MCC), supramarginal gyrus (SMG), and postcentral gyrus (PoCG). Using the GCA algorithm, we detected abnormal effective connectivity (EC) in the inferior occipital gyrus (IOG), MCC, Cerebelum_Crus1, and PoCG. Furthermore, correlations between disrupted FC/EC and clinical characteristics, especially tinnitus distress-related characteristics, were found in AT patients.

Conclusions: Our work demonstrated abnormal FC and EC between the frontostriatal network and several nonauditory regions in AT patients with hearing loss, suggesting that multiple large-scale network dysfunctions and interactions are involved in the perception of tinnitus. These findings not only enhance the current understanding of the frontostriatal network in tinnitus but also serve as a reminder of the importance of focusing on tinnitus at an early stage.

Keywords: Functional connectivity, Frontostriatal Network, Granger causality analysis, Resting-state fMRI, Tinnitus 


\section{Introduction}

Tinnitus is a type of sensory-perceptual disorder that is evident in the absence of an external sound source and affects millions of people (Y Cai et al., 2018; Vanneste, Alsalman, \& De Ridder, 2019). It is estimated that approximately $10-15 \%$ of the adult population has tinnitus, and the incidence can reach 30\% depending on age (J. P. Rauschecker, May, Maudoux, \& Ploner, 2015; Yang et al., 2018). People who have tinnitus often experience depression, anxiety and sleep disturbances, which may reduce their quality of life (Baguley, McFerran, \& Hall, 2013; Henry, Roberts, Caspary, Theodoroff, \& Salvi, 2014). Different management strategies have been recommended for tinnitus, including psychological counseling, cognition therapy, and sound therapy; however, there is no definitive cure for all types of tinnitus (Chen et al., 2017; Tsai et al., 2018). Additionally, the neurological mechanisms of tinnitus remain unclear, and the underlying causes have not yet been elucidated. It is widely accepted that the central nervous system (CNS) is believed to play an important role in the pathophysiology of tinnitus (Yuexin Cai et al., 2017; Chen et al., 2016).

Numerous neuroimaging studies have shown that nonauditory systems, especially the limbic system, make important contributions to the evaluation and modulation of tinnitus perception signals (Barry, Paolini, Robertson, \& Mulders, 2015; Gunbey et al., 2017; J. Rauschecker, Leaver, \& Mühlau, 2010; J. P. Rauschecker et al., 2015). Decreased GMV has been reported in the ventromedial and dorsomedial prefrontal cortices (vmPFC and dmPFC, respectively), nucleus accumbens (NAc), anterior cingulate cortex (ACC), posterior cingulate cortex, hippocampus and supramarginal gyrus (A. Leaver et al., 2012; Mahoney et al., 2011). Another study showed disrupted core areas of the NAc and vmPFC, which may lead to the occurrence of tinnitus (J. P. Rauschecker et al., 2015). The NAc is a subcortical structure that is located at the base of the forebrain and is a component of the ventral striatum (Cauda et 
al., 2011). Its best known functional role is as a component of the mesolimbic pathway, which is responsible for the reward system together with the ventral tegmental area (Lammel et al., 2012; Navratilova et al., 2012). A few studies have further revealed that the NAc has multiple functions, including sensory gating, motivation, reinforcement learning, and salience (D De Ridder et al., 2016; Koechlin, 2016; Takahashi et al., 2017). Past studies have linked the role of NAc to the pathology of tinnitus and suggested that the vmPFC/subgenual ACC ( $\mathrm{sgACC}$ ) and NAc may form a frontostriatal network (A. Leaver et al., 2016; Amber M. Leaver et al., 2011). This network is responsible for assigning affective meaning or subjective value to sensory signals and, ultimately, for eliciting action to avoid negative outcomes (J. P. Rauschecker et al., 2015).

The tinnitus symptoms can be acute or chronic. In clinical practice, we noticed that acute tinnitus (AT) patients are much more eager to seek for clinical help than chronic tinnitus patients. Few studies have investigated frontostriatal circuits in AT patients (Y. Cai et al., 2020). Thus, the mechanism underlying this network remains unclear. It is important to reveal the abnormalities of the frontostriatal network of AT to prevent the transition from acute to chronic tinnitus. The present study aimed to increase our understanding of the mechanism of the frontostriatal network in the context of AT. Resting-state fMRI (rs-fMRI) has proven to be a useful tool for investigating intrinsic neuro-pathophysiological mechanisms in tinnitus by detecting abnormal brain functional connectivity (FC) and neural activity (Chen, Chen, et al., 2018; Chen, Zhang, et al., 2018). Based on the potential role within the frontostriatal network in tinnitus, the bilateral NAc and vmPFC were selected as seed regions. We hypothesized that the AT patients would show an altered connectivity in the NAc/vmPFC by utilizing the FC method. Additionally, the Granger causality analysis (GCA) method has been widely used to study effective connectivity (EC) in normal brains and some other neurological or 
psychiatric disorders, such as depression, mild cognitive impairment, Alzheimer's disease, and presbycusis (Chen et al., 2020; Guo et al., 2015; Yu et al., 2017). GCA was used to analyze directionality in disrupted EC between the NAc and the vmPFC among subjects. In the current study, we hypothesized that a disruption of the EC between the NAc and vmPFC would be recognized by using the GCA method. We further hypothesized that disrupted frontostriatal connectivity would be associated with tinnitus-related characteristics. 


\section{Materials and methods}

\section{Subjects}

In this study, two groups of subjects were included, the AT patient group and healthy control (HC) group, with a total of 85 right-handed people. The two groups were age-, gender-, and education-matched. The selection criteria for AT patients were as follows: constant, unilateral subjective tinnitus with sensorineural hearing loss (SNHL) in one ear with a duration less than 1 month. There were 18 right-side and 24 left-side tinnitus patients in the AT group ( 24 females). The HC group included 43 subjects (27 females). The hearing level was determined by pure-tone audiometry (PTA) at frequencies of 125, 250, 500, 1000, 2000, 4000 and $8000 \mathrm{~Hz}$. All patients had hearing loss of more than $30 \mathrm{~dB}$ in one ear at frequencies from 0.125 to $8 \mathrm{kHz}$, whereas the $\mathrm{HC}$ group subjects had no hearing loss at any of the 7 frequencies (hearing thresholds $<25 \mathrm{~dB}$ ). Additionally, the exclusion criteria for this study were the same as described in our previous research (Zhou et al., 2019). The details are as follows: pulsatile tinnitus; Meniere's disease; hyperacusis; neurological or psychiatric illness, such as Alzheimer's disease, Parkinson's disease, epilepsy, and major depression; head injury; major medical illness (e.g., cancer, anemia, and thyroid dysfunction); and MRI contraindications.

Moreover, three questionnaires were used in the study: The Tinnitus Handicap Inventory (THI), the Self-Rating Depression Scale (SDS) and the Self-Rating Anxiety Scale (SAS). THI, a self-reported tinnitus handicap questionnaire, was applied to estimate the severity of tinnitus and related distress (Meng et al., 2012). The SDS and SAS were administered to all subjects to exclude depression or anxiety. None of the participants experienced an anxiety or depressive state (overall scores $<50$ for each)(W. W. Zung, 1971; W. W. K. Zung, 1986). All participants' demographics and clinical features are presented in Table 1. 


\section{MRI data acquisition}

All MR scans were performed on a 3.0 T Philips system (Ingenia, The Netherlands) with an 8-channel receive-only head coil. We used headphones and sponge pads to minimize scanner noise and head movement. During the MR acquisition, all participants were asked to remain still with their eyes closed but to stay awake and not think about anything in particular. First, high-resolution structural images were obtained using a coronal T1-weighted three-dimensional turbo fast echo (3D-TFE) sequence with the following parameters: echo time $(\mathrm{TE})=3.7 \mathrm{~ms}$ and repetition time $(\mathrm{TR})=8.1 \mathrm{~ms} ; 170$ slices; 1 -mm slice thickness; 0-mm interslice gap; 8-degree flip angle (FA); 256×256 matrix; and field of view (FOV) of $256 \mathrm{~mm} \times 256 \mathrm{~mm}$. Based on the structural images, the rs-fMRI images were acquired by a gradient echo-planar imaging sequence as follows: $\mathrm{TR}=2000 \mathrm{~ms}$ and $\mathrm{TE}=30 \mathrm{~ms} ; 36$ slices; 4-mm slice thickness; 0-mm interslice gap; 90-degree FA; $64 \times 64$ matrix; FOV of $240 \mathrm{~mm} \times 240 \mathrm{~mm}$; and 230 volumes. The acquisition times of the structural and fMRI sequences were 5 minutes 29 seconds and 8 minutes 8 seconds, respectively.

\section{MRI data analyses}

\section{Data preprocessing}

Data analyses were performed using the toolbox for Data Processing \& Analysis for Brain Imaging (DPABI V4.2; http://rfmri.org/dpabi), which is based on Statistical Parametric Mapping (SPM8). First, the first 10 volumes were removed to reduce deviation. Then, the remaining data were slice-timing corrected (with the middle slice as the reference slice) and realigned for motion correction. The generated images underwent spatial normalization by using the Montreal Neurological Institute (MNI) template (resampling voxel size $=3 \mathrm{~mm} \times 3 \mathrm{~mm} \times 3 \mathrm{~mm}$ ) and were then smoothed using an isotropic 
Gaussian kernel (full width at half maximum $=6 \mathrm{~mm}$ ). Then, removal of the linear trend of the time courses and temporal bandpass filtering $(0.01-0.08 \mathrm{~Hz})$ were performed. In addition, we regressed out nuisance covariates, including head motion profiles (Friston 24-parameter model) (Friston, Williams, Howard, Frackowiak, \& Turner, 1996), white matter signals, and cerebrospinal fluid signals. Any subjects who had a head motion greater than $2.0 \mathrm{~mm}$ or rotation greater than $2.0^{\circ}$ were excluded from the study.

\section{Seed-based functional connectivity analysis}

A seed-based FC analysis was performed to analyze the data using predefined seeds in our study. The time series of each region of interest (ROI) was defined as the average time series across all voxels in the seed region. For each ROI, seed-based FC was computed as Pearson correlation coefficients between the seeds and the whole brain in a voxel-wise manner. Then, an FC map was acquired for each subject. The FC map was standardized to a zFC map using Fisher's z-transformation.

We defined two 5-mm-radius spherical ROIs for the bilateral NAc using center coordinates obtained from the literature as well as the bilateral vmPFC. Their respective MNI space coordinates can be seen in Table S1. We used these ROIs as seed regions for the whole-brain FC analysis, controlling for the effects of age, sex, education and GM volume using the DPABI toolbox. Then, comparison between the two groups (tinnitus > controls) was performed. A Gaussian random field (GRF) correction was used for multiple comparison correction (voxel-level $\mathrm{p}<0.005$, cluster-level $\mathrm{p}<0.05$ ).

\section{Granger causality analysis}

GCA patterns were evaluated using the rs-fMRI Data Analysis Toolkit plus (RESTplus, 
http://restfmri.net/forum/). The voxel-wise GCA was conducted to estimate the EC of the ROIs of the bilateral NAc/vmPFC $(\mathrm{X})$ on every other voxel in the brain $(\mathrm{Y})$. A positive coefficient from $\mathrm{X}$ to $\mathrm{Y}$ suggested a linear direct influence of region $\mathrm{X}$ on region $\mathrm{Y}$, while a negative coefficient from $\mathrm{X}$ to $\mathrm{Y}$ indicated that the region Y exerts a causal influence on the activity region X. For each ROI, EC maps were computed as Pearson correlation coefficients (r) between the seeds and the whole brain in a voxel-wise manner. Then, a Fisher z-transformation was used to convert all Pearson correlation (r) values to z-scores. For group-level comparison, clusters passing a GRF correction with voxel-level $p<$ 0.005 and cluster-level $\mathrm{p}<0.05$ were considered significant (age, sex, GM volume and education were considered covariates).

Then, the ROI-wise GCA data to detect EC within the bilateral NAc and vmPFC. For these 6 pairs (left NAc and right NAc, left NAc and left vmPFC, left NAc and right vmPFC, right NAc and left vmPFC, right NAc and right vmPFC, and left vmPFC and right vmPFC), we extracted time series of the brain regions $(\mathbf{X}, \mathbf{Y})$. Signed path coefficients were then calculated as a GCA value, including $\mathbf{X} \rightarrow \mathbf{Y}$ and $\mathbf{Y} \rightarrow \mathbf{X}$. Furthermore, two-sample $\mathrm{t}$ tests were used for the GCA value to determine the differences between the two groups. The significance level was set at $\mathrm{p}<0.05$.

\section{Statistical analysis}

For the between-group differences in demographic and clinical characteristics, independent two-sample t-tests were used for age, education, PTA, duration, and THI, and the $\chi^{2}$ test was applied for gender. The SPSS software version 22 was used for the independent samples $t$ tests and $\chi^{2}$ test $(p<0.05$ was considered to indicate significance). Shapiro-Wilk tests were used to evaluate data normality, and $p>$ 0.05 indicated a normal distribution of data. 
Pearson's correlation analysis was used to study the correlation between the clinical features and the abnormal FC/GCA in AT patients. Furthermore, partial correlations were applied between these connections and duration/THI/SDS/SAS, controlling for the effects of age, sex, education and mean hearing loss. We assessed all correlations and partial correlations for significance at the 0.05 level. 


\section{Results}

\section{Demographic and clinical information}

The AT group showed no significant differences in age, sex, or educational level compared with the HC group. During the auditory test, tinnitus patients exhibited unilateral hearing loss, with a significant difference from that of $\mathrm{HC}$ subjects $(\mathrm{p}<0.05)$. Additionally, patients in the AT group demonstrated higher SAS and SDS scores than subjects in the HC group $(\mathrm{p}<0.05)$. The specific information is shown in Table 1.

\section{Seed-based FC analysis}

Compared with the subjects in the HC group, patients in the AT group showed significantly decreased connectivity between the seed region in the left NAc and the left orbitofrontal cortex (OFC) and the left midcingulate cortex (MCC) (Table 2 and Figure 1A). In addition, the right NAc showed reduced connectivity with the right middle frontal gyrus (MFG), the right supramarginal gyrus (SMG) and the right postcentral gyrus (PoCG) in AT patients (Table 2 and Figure 1B).

When we designated an ROI in the left vmPFC, reduced FC was found in the right superior frontal gyrus (SFG) in tinnitus patients compared with that in $\mathrm{HC}$ subjects (Table 2 and Figure 1C). Moreover, we also found reduced connectivity between the right vmPFC and the right MFG and the SFG in AT patients (Table 2 and Figure 1D). We did not find any increased FC in the voxel-wise analysis. Moreover, the significant differences noted in brain regions in the seed-based FC analysis were mainly located outside the auditory areas.

\section{GCA findings}


The voxel-wise GCA findings are illustrated in Table 3 and Figure 2. Compared with HC subjects, patients with AT revealed significantly decreased EC from the left NAc to the left inferior occipital gyrus (IOG) (Table 3 and Figure 2). Additionally, significantly reduced EC from the right NAc to the left MCC was also observed in AT patients compared with that in HC subjects (Table 3 and Figure 2). However, the right vmPFC demonstrated a lower causal influence on the left Cerebelum_Crus1 (Table 3 and Figure 2). No significantly different EC was found from the left vmPFC to the entire brain.

Compared with the connectivity in the HC group, we found enhanced EC from the bilateral MCC to the left NAc in AT patients (Table 3 and Figure 2). In addition, the left MCC and the left PoCG showed increased EC with the right NAc (Table 3 and Figure 2). Interestingly, we did not detect any significantly EC from the whole brain to the bilateral vmPFC in the study. However, we did not find any significant EC within the bilateral NAc and vmPFC.

\section{Correlation analysis}

The reduced FC between the left NAc and the left OFC exhibited a significant positive correlation with THI scores $(r=0.343, p=0.033)$, as shown in Table S2 and Figure 1E. Moreover, Pearson's correlation analyses revealed a positive correlation between duration and enhanced EC from the left MCC to the right NAc $(r=0.372, p=0.020)$ (Table S3 and Figure 3B). In addition, SDS scores and SAS scores correlated positively with decreased EC from the right vmPFC and the left Cerebelum_Crus1 $(r=0.319, p=0.047 ; r=0.395, p=0.013)$ (Table S3 and Figure 3A/3C). These correlations were assessed after correcting for the effects of age, gender, education, and mean hearing level. Furthermore, we performed non-adjusted correlation analyses without controlling for demographic factors, and it showed same patterns with partial correlation analyses (Table S4, S5). 


\section{Discussion}

The present study combined FC and GCA methods to explore the aberrant frontostriatal network in unilateral AT patients with hearing loss. We selected the NAc and vmPFC as seed regions, as the NAc and $\mathrm{vmPFC}$ may participate in a frontostriatal gating circuit according to previous studies. Our FC analysis revealed significantly decreased FC between these seed regions and several nonauditory regions, including the prefrontal cortices, SMG, MCC and PoCG. Based on the GCA method, we also detected abnormal EC between the ROIs in the frontostriatal network and several brain regions outside the auditory area, including the PoCG, MCC, Cerebelum_Crus1. Moreover, these altered connectivities correlated with the severity of tinnitus distress. Our study clearly demonstrated abnormal functional and causal connectivity between the frontostriatal network and nonauditory regions in patients with unilateral AT with hearing loss.

In our study, the AT patients showed a general reduction in FC between the seed regions in the frontostriatal network and the prefrontal cortices, principally the OFC, MFG, and SFG. Our findings indicated that the frontal cortex plays a crucial role in the mechanism of tinnitus. A majority of previous neuroimaging studies have confirmed the contribution of the frontal cortex to tinnitus (Burton et al., 2012; Chen et al., 2016; Chen et al., 2015; Chen et al., 2014; S. A. Schmidt, K. Akrofi, J. R. Carpenter-Thompson, \& F. T. Husain, 2013). In addition, a model was developed to demonstrate that the prefrontal cortex may contribute to certain perceptual features of tinnitus (J. Rauschecker et al., 2010). Our work also demonstrated decreased connectivity between the NAc and MFG and between the NAc and the OFC. The vmPFC is an overlapping area of the OFC and MFG. Thus, these findings reflect an interwoven relationship between the NAc and the vmPFC in AT patients, which is in accordance with the results in a study identifying those regions as ROIs (Cauda et al., 2011). Furthermore, our correlation analyses revealed that the reduced FC between the NAc and OFC 
correlated positively with tinnitus distress. These results provide direct evidence that frontostriatal circuits participate in AT. In contrast, a few researchers (Amber M. Leaver et al., 2011; A. M. Leaver, Seydell-Greenwald, \& Rauschecker, 2016; J. P. Rauschecker et al., 2015) reported increased frontostriatal connectivity in chronic tinnitus patients, whereas we found a reduction in FC between the NAc and prefrontal areas. Similarly, a recent study that focused on AT showed reduced FC between the NAc and SFG (Y. Cai et al., 2020). On the other hand, some studies have reported increased NAcvmPFC FC in patients, which demonstrated a causal relationship between frontostriatal circuits and the transition from acute to chronic pain. Considering the well-established similarities between chronic pain and tinnitus (D. De Ridder, Elgoyhen, Romo, \& Langguth, 2011; Møller, 2007; J. P. Rauschecker et al., 2015), we speculate that the reduction in FC of the frontostriatal network may reflect the early stage of tinnitus, and it progresses into increased connectivity as tinnitus transforms from acute to chronic state. However, the relationship between acute and chronic tinnitus remains unclear and needs future research based on longitudinal data for verification.

FC and GCA analyses both revealed a strong interactive relationship between the NAc and MCC, a key region connected to circuits mediating cognitive control, nociceptive perception and multisensory integration (Hu et al., 2019; Ospina, Jalilianhasanpour, \& Perez, 2019). Vogt (2016) suggested a functional MCC circuit in which the anterior MCC was associated with fear, fear-associated memories and cognitive schemata. In addition, the MCC is a key region of the frontoparietal-limbic network, which correlates with emotional processing (Golm, Schmidt-Samoa, Dechent, \& Kroner-Herwig, 2013). A recent study has also reported that tinnitus distress correlates with cortical thickness and cortical surface area in the bilateral cingulate cortex (Meyer et al., 2016). Furthermore, the MCC is a hub in the salience network (a vital large-scale intrinsic network responsible for perception). The MCC has also 
been reported to integrate stimuli from the auditory pathway (Heywood et al., 2017). In patients with auditory hallucinations, the activity of the substantia nigra was disturbed, suggesting greater relevance and attention to internally generated auditory perception (Lefebvre et al., 2016; Sommer et al., 2008). In the current study, we found increased effective FC from the MCC to the NAc, and tinnitus duration was positively corrected with this increased effective FC. Consequently, our findings provided evidence that multiple large-scale network dysfunctions and interactions were involved in the perception of tinnitus.

Our study also revealed disrupted EC from the vmPFC to the cerebellum. The cerebellum is a key hub responsible for detecting auditory input and sound processing (Zhou et al., 2019) and is considered to play an important role in gating control systems (Chen et al., 2015). Our study revealed decreased EC between the vmPFC and the cerebellum. Furthermore, the SDS and SAS scores correlated positively with this decreased EC. Our finding is consistent with that in our previous study (Zhou et al., 2019) and recent studies focused on AT and the frontostriatal network (Y. Cai et al., 2020; Hullfish, Abenes, Yoo, De Ridder, \& Vanneste, 2019). The cerebellum can also receive and process information input from auditory regions (Petacchi, Laird, Fox, \& Bower, 2005). In addition, disrupted connectivity between the auditory cortex and the cerebellum was found in tinnitus patients (Maudoux et al., 2012). Therefore, the abnormal EC between the cerebellum and vmpFC may lead to a reduced capability to distinguish and integrate afferent signals.

Moreover, aberrant FC and EC in the PoCG and SMG was found in patients with AT. The PoCG is a crucial region of the somatosensory system. In patients experiencing a loss of auditory input, the integration of somatosensory and auditory system is damaged (Froemke \& Martins, 2011), which is consistent with our finding of a decreased FC between the NAc and PoCG in AT patients with hearing 
loss. Therefore, in addition to the alteration of the peripheral auditory system, the dysfunction of the somatosensory system may be responsible for tinnitus (Job et al., 2012). The somatosensory system delivers auditory information to the NAc directly; thus, the emotional content of the sound could be evaluated. The NAc was also the key hub of the noise canceling system (Song, Vanneste, \& De Ridder, 2015). Unwanted sounds will be filtered out by this system, such that these sounds do not reach auditory perception. Our study indicated that the information flow from the somatosensory system to the NAc will be damaged after a complete loss of the peripheral auditory system and it may compromise the noise cancelling system. As a result, the aberrant sound related to tinnitus may reach the cortex, leading to its conscious perception in AT patients. The SMG is a region associated with the attention network (Yamasaki, LaBar, \& McCarthy, 2002), and we found reduced FC between the NAc and SMG in AT patients. Based on EEG and PET, previous studies also suggested a possible role for SMG in tinnitus (Mirz et al., 1999; Weiler, Brill, Tachiki, \& Wiegand, 2000). Based on seed-FC analysis, tinnitus subjects showed a diminished relationship between the dorsal attention network (DAN) and the right SMG (S. Schmidt, K. Akrofi, J. Carpenter-Thompson, \& F. J. P. o. Husain, 2013). Thus, the reduced FC in the SMG may change the connectivity between the DAN and the frontostriatal network in tinnitus patients.

Several limitations of this study should be noted. Our study was based on a limited sample size and a cross-sectional study design, so the results should be interpreted with caution. Given the high heterogeneity in AT patients, such as laterality and hearing loss, the influence of heterogeneity still exists even when applying strict criteria for inclusion and exclusion. Thus, future studies that involve more subjects and subgroups using a longitudinal study design are required. Moreover, although we applied earphones to reduce noise effects in our study, the scanner noise could not be completely 
prevented, which may have influenced the neural activity to a certain degree during resting-state measurements. 


\section{Conclusions}

By combining FC and GCA, our work demonstrated abnormal functional and causal connectivity between the frontostriatal network and several nonauditory regions in AT patients with hearing loss. These findings not only enhance the current understanding of the frontostriatal network in tinnitus populations but also provide evidence that multiple large-scale network dysfunctions and interactions are involved in the perception of tinnitus. Furthermore, the present work emphasizes the importance of paying close attention to this acute hearing disorder. 
Acknowledgments

None 


\section{Declarations}

Funding This work was supported by the Youth Medical Talents of Jiangsu Province (No.

QNRC2016062), 14th “Six Talent Peaks” Project of Jiangsu Province (No. YY-079), Nanjing

Outstanding Youth Fund (No. JQX17006), and 333 High-level Talents Training Project of Jiangsu

Province (No. BRA2019122).

Conflict of interest The authors declare no conflict of interest.

Ethical approval The present study was approved by the Research Ethics Committee of Nanjing

Medical University.

Consent to participate All subjects provided written informed consent prior to participation in the study.

Consent for publication Written informed consent for publication was obtained from all participants.

Availability of data and material The datasets used or analysed during the current study are available from the corresponding author on reasonable request.

Code availability Not applicable.

Authors' contributions Gang-Ping Zhou and Yu-Chen Chen designed the experiment, collected the data, performed the analysis, and wrote the manuscript. Wang-Wei Li, Heng-Le Wei, Yu-Sheng Yu, and Qing-Qing Zhou helped collect the data. Xindao Yin helped perform the analysis. Hong Zhang and Yue-Jin Tao contributed to the discussion and manuscript revision. All authors have read and approved the manuscript. 


\section{Figure legends}

Figure 1 (A-D) The significant FC between the bilateral NAc/vmPFC seed regions and the whole brain (GRF correction, voxel-level $\mathrm{p}<0.005$, cluster-level $\mathrm{p}<0.05$ ). (E) The THI scores were positively correlate with the reduced FC between the left NAc and the left OFC ( $r=0.343, p=0.033)$. L, left; R, right; B. bilateral. Blue means decreased connectivity. The color bar represents the t-value. 
Figure 2 (D-F) The EC of the NAc and vmPFC. The blue line represents the EC from the bilateral $\mathrm{NAc} / \mathrm{vmPFC}$ to the other brain regions; the red line means the $\mathrm{EC}$ from the other brain regions to the bilateral NAc/vmPFC. (A) and (C) The SDS scores and the SAS scores were positively correlate with the decreased effective connectivity from the right vmPFC and the left Cerebelum_Crus1 $(r=0.319, p$ $=0.047 ; \mathrm{r}=0.395, \mathrm{p}=0.013$ ). (B) Positive correlation between the enhanced effective connectivity from the left MCC to the right NAc $(r=0.372, p=0.020)$. 


\section{Tables}

Table 1 Demographic and clinical characteristics of participants

\begin{tabular}{cccc}
\hline & ATs $(\mathrm{n}=42)$ & HCs $(\mathrm{n}=43)$ & P value \\
\hline Age(years) & $43.24 \pm 12.32$ & $46.93 \pm 13.33$ & 0.189 \\
Genger(male/female) & $18 / 24$ & $16 / 27$ & 0.60 \\
Education(years) & $12.10 \pm 2.93$ & $12.44 \pm 3.25$ & 0.61 \\
Handedness(right/left) & $42 / 0$ & $43 / 0$ & 1.00 \\
Tinnitus laterality(right/left) & $18 / 24$ & - & - \\
Duration(days) & $7.48 \pm 3.09$ & - & - \\
PTA of right ear $(\mathrm{dB})$ & $59.36 \pm 12.38$ & $14.32 \pm 2.43$ & $<.001^{*}$ \\
PTA of left ear $(\mathrm{dB})$ & $58.48 \pm 13.24$ & $14.45 \pm 2.56$ & $<0.001^{*}$ \\
THI score & $33.02 \pm 8.82$ & - & - \\
SAS score & $31.43 \pm 5.06$ & $25.53 \pm 2.66$ & $<0.001^{*}$ \\
SDS score & $30.60 \pm 6.09$ & $25.72 \pm 1.96$ & $<0.001 *$ \\
\hline
\end{tabular}

Data are presented as mean \pm SD. PTA, Pure-tone audiometry; THI, Tinnitus Handicap Inventory; SAS, Self-Rating Anxiety Scale; SDS, Self-Rating Depression Scale.

*P $<0.001$ (independent-sample t-test, two-tailed), showed statistical difference in hearing threshold between ATs and HCs. 
Table 2 The significant FC between the bilateral NAc/vmPFC seed regions and the whole brain

\begin{tabular}{|c|c|c|c|c|}
\hline \multirow[t]{2}{*}{ Seed region } & \multirow[t]{2}{*}{ Brain region } & $\begin{array}{l}\text { Peak MNI } \\
\text { coordinates }\end{array}$ & \multirow[t]{2}{*}{ T score } & \multirow{2}{*}{$\begin{array}{c}\text { Cluster Size } \\
\text { (voxels) }\end{array}$} \\
\hline & & $(\mathrm{x}, \mathrm{y}, \mathrm{z})$ & & \\
\hline \multirow[t]{2}{*}{ L. NAc } & L. Orbitofrontal cortex & $-12,42,-18$ & -3.6812 & 70 \\
\hline & B. Midcingulate cortex & $15,-12,45$ & -4.056 & 122 \\
\hline \multirow[t]{3}{*}{ R. NAc } & R. Middle Frontal gyrus & $6,24,-9$ & -3.6649 & 138 \\
\hline & R. Supramarginal gyrus & $66,-42,27$ & -3.5207 & 80 \\
\hline & R. Postcentral gyrus & $24,-45,75$ & -3.7798 & 119 \\
\hline L. vmPFC & R. Superior frontal gyrus & $18,15,63$ & -4.1989 & 187 \\
\hline \multirow[t]{2}{*}{ R. vmPFC } & R. Middle frontal gyrus & $36,60,21$ & -3.7313 & 63 \\
\hline & R. Superior frontal gyrus & $18,15,63$ & -4.6636 & 313 \\
\hline
\end{tabular}

GRF correction was used for multiple comparisons correction (voxel-level $p<0.005$, cluster-level $\mathrm{p}<$ 0.05). L, Left; R, Right; B, Bilateral; MNI, Montreal Neurological Institute. 
Table 3 Abnormal effective connectivity between the bilateral NAc/vmPFC and the whole brain

\begin{tabular}{|c|c|c|c|c|}
\hline & \multirow[t]{2}{*}{ Brain region } & $\begin{array}{c}\text { Peak MNI } \\
\text { coordinates }\end{array}$ & \multirow[t]{2}{*}{ T score } & \multirow{2}{*}{$\begin{array}{c}\text { Cluster Size } \\
\text { (voxels) }\end{array}$} \\
\hline & & $(\mathrm{x}, \mathrm{y}, \mathrm{z})$ & & \\
\hline From L. NAc & L. Inferior occipital gyrus & $-24,-93,-6$ & -4.4009 & 89 \\
\hline To L. NAc & B. Midcingulate cortex & $6,0,36$ & 4.1399 & 87 \\
\hline From R. NAc & L. Midcingulate cortex & $-9,-30,51$ & -3.8987 & 82 \\
\hline \multirow[t]{2}{*}{ To R. NAc } & L. Midcingulate cortex & $-9,-30,48$ & 4.711 & 95 \\
\hline & L. Postcentral gyrus & $-33,-39,54$ & 4.0026 & 65 \\
\hline From R. vmPFC & L. Cerebelum_Crus1 & $-6,-81,-18$ & -3.8133 & 58 \\
\hline
\end{tabular}

GRF correction was used for multiple comparisons correction (voxel-level $p<0.005$, cluster-level $\mathrm{p}<$ 0.05). L, Left; R, Right; B, Bilateral; MNI, Montreal Neurological Institute. 


\section{References}

Baguley, D., McFerran, D., \& Hall, D. (2013). Tinnitus. Lancet, 382(9904), 1600-1607. doi:10.1016/s0140-6736(13)60142-7

Barry, K., Paolini, A., Robertson, D., \& Mulders, W. J. N. (2015). Modulation of medial geniculate nucleus neuronal activity by electrical stimulation of the nucleus accumbens. 308, 1-10. doi:10.1016/j.neuroscience.2015.09.008

Burton, H., Wineland, A., Bhattacharya, M., Nicklaus, J., Garcia, K. S., \& Piccirillo, J. F. (2012). Altered networks in bothersome tinnitus: a functional connectivity study. BMC Neurosci, 13, 3 . doi:10.1186/1471-2202-13-3

Cai, Y., Huang, D., Chen, Y., Yang, H., Wang, C., Zhao, F., . . Zheng, Y. J. F. i. b. n. (2018). Deviant Dynamics of Resting State Electroencephalogram Microstate in Patients With Subjective Tinnitus. 12, 122. doi:10.3389/fnbeh.2018.00122

Cai, Y., Xie, M., Su, Y., Tong, Z., Wu, X., Xu, W., . . Zheng, Y. (2020). Aberrant Functional and Causal Connectivity in Acute Tinnitus With Sensorineural Hearing Loss. Front Neurosci, 14, 592. doi:10.3389/fnins.2020.00592

Cai, Y., Zhou, Q., Yang, H., Jiang, J., Zhao, F., Huang, X., . . Zheng, Y. (2017). Logistic regression analysis of factors influencing the effectiveness of intensive sound masking therapy in patients with tinnitus. 7(11), e018050. doi:10.1136/bmjopen-2017-018050\%J BMJ Open

Cauda, F., Cavanna, A., D'agata, F., Sacco, K., Duca, S., \& Geminiani, G. J. J. o. c. n. (2011). Functional connectivity and coactivation of the nucleus accumbens: a combined functional connectivity and structure-based meta-analysis. 23(10), 2864-2877. doi:10.1162/jocn.2011.21624

Chen, Y. C., Chen, H., Bo, F., Xu, J. J., Deng, Y., Lv, H., . . L Lu, G. (2018). Tinnitus distress is associated with enhanced resting-state functional connectivity within the default mode network. Neuropsychiatr Dis Treat, 14, 1919-1927. doi:10.2147/ndt.S164619

Chen, Y. C., Feng, Y., Xu, J. J., Mao, C. N., Xia, W., Ren, J., \& Yin, X. (2016). Disrupted Brain Functional Network Architecture in Chronic Tinnitus Patients. Front Aging Neurosci, 8, 174. doi:10.3389/fnagi.2016.00174

Chen, Y. C., Wang, F., Wang, J., Bo, F., Xia, W., Gu, J. P., \& Yin, X. (2017). Resting-State Brain Abnormalities in Chronic Subjective Tinnitus: A Meta-Analysis. Front Hum Neurosci, 11, 22. doi:10.3389/fnhum.2017.00022

Chen, Y. C., Xia, W., Feng, Y., Li, X., Zhang, J., Feng, X., . . . Teng, G. J. (2015). Altered interhemispheric functional coordination in chronic tinnitus patients. Biomed Res Int, 2015, 345647. doi:10.1155/2015/345647

Chen, Y. C., Yong, W., Xing, C., Feng, Y., Haidari, N. A., Xu, J. J., . . Wu, Y. (2020). Directed functional connectivity of the hippocampus in patients with presbycusis. Brain Imaging Behav, 14(3), 917-926. doi:10.1007/s11682-019-00162-z

Chen, Y. C., Zhang, H., Kong, Y., Lv, H., Cai, Y., Chen, H., . . Yin, X. (2018). Alterations of the default mode network and cognitive impairment in patients with unilateral chronic tinnitus. Quant Imaging Med Surg, 8(10), 1020-1029. doi:10.21037/qims.2018.11.04

Chen, Y. C., Zhang, J., Li, X. W., Xia, W., Feng, X., Gao, B., . . . Teng, G. J. (2014). Aberrant spontaneous brain activity in chronic tinnitus patients revealed by resting-state functional MRI. Neuroimage Clin, 6, 222-228. doi:10.1016/j.nicl.2014.09.011 
De Ridder, D., Elgoyhen, A. B., Romo, R., \& Langguth, B. (2011). Phantom percepts: tinnitus and pain as persisting aversive memory networks. Proc Natl Acad Sci U S A, 108(20), 8075-8080. doi:10.1073/pnas.1018466108

De Ridder, D., Vanneste, S., Gillett, G., Manning, P., Glue, P., \& Langguth, B. J. N. j. o. t. I. N. S. (2016). Psychosurgery Reduces Uncertainty and Increases Free Will? A Review. 19(3), 239-248. doi:10.1111/ner.12405

Friston, K. J., Williams, S., Howard, R., Frackowiak, R. S., \& Turner, R. (1996). Movement-related effects in fMRI time-series. Magn Reson Med, 35(3), 346-355. doi:10.1002/mrm.1910350312

Froemke, R. C., \& Martins, A. R. (2011). Spectrotemporal dynamics of auditory cortical synaptic receptive field plasticity. Hear Res, 279(1-2), 149-161. doi:10.1016/j.heares.2011.03.005

Golm, D., Schmidt-Samoa, C., Dechent, P., \& Kroner-Herwig, B. (2013). Neural correlates of tinnitus related distress: an fMRI-study. Hear Res, 295, 87-99. doi:10.1016/j.heares.2012.03.003

Gunbey, H., Gunbey, E., Aslan, K., Bulut, T., Unal, A., \& Incesu, L. J. C. n. (2017). Limbic-Auditory Interactions of Tinnitus: An Evaluation Using Diffusion Tensor Imaging. 27(2), 221-230. doi:10.1007/s00062-015-0473-0

Guo, W., Liu, F., Zhang, Z., Liu, J., Yu, M., Zhang, J., . . . Zhao, J. (2015). Unidirectionally affected causal connectivity of cortico-limbic-cerebellar circuit by structural deficits in drug-naive major depressive disorder. $J$ Affect Disord, 172, 410-416. doi:10.1016/j.jad.2014.10.019

Henry, J., Roberts, L., Caspary, D., Theodoroff, S., \& Salvi, R. J. J. o. t. A. A. o. A. (2014). Underlying mechanisms of tinnitus: review and clinical implications. 25(1), 5-22; quiz 126. doi:10.3766/jaaa.25.1.2

Heywood, R., Gao, Q., Nyunt, M., Feng, L., Chong, M., Lim, W., . . . disorders, g. c. (2017). Hearing Loss and Risk of Mild Cognitive Impairment and Dementia: Findings from the Singapore Longitudinal Ageing Study. 43, 259-268. doi:10.1159/000464281

Hu, T., Wang, R., Du, Y., Guo, F., Wu, Y., Wang, Y., . . Chen, Z. J. T. J. o. n. t. o. j. o. t. S. f. N. (2019). Activation of the Intrinsic Pain Inhibitory Circuit from the Midcingulate $\mathrm{Cg} 2$ to Zona Incerta Alleviates Neuropathic Pain. 39(46), 9130-9144. doi:10.1523/jneurosci.1683-19.2019

Hullfish, J., Abenes, I., Yoo, H. B., De Ridder, D., \& Vanneste, S. (2019). Frontostriatal network dysfunction as a domain-general mechanism underlying phantom perception. Hum Brain Mapp, 40(7), 2241-2251. doi:10.1002/hbm.24521

Job, A., Pons, Y., Lamalle, L., Jaillard, A., Buck, K., Segebarth, C., \& Delon-Martin, C. (2012). Abnormal cortical sensorimotor activity during "Target" sound detection in subjects with acute acoustic trauma sequelae: an fMRI study. Brain Behav, 2(2), 187-199. doi:10.1002/brb3.21

Koechlin, E. J. C. o. i. n. (2016). Prefrontal executive function and adaptive behavior in complex environments. 37, 1-6. doi:10.1016/j.conb.2015.11.004

Lammel, S., Lim, B., Ran, C., Huang, K., Betley, M., Tye, K., . . . Malenka, R. J. N. (2012). Input-specific control of reward and aversion in the ventral tegmental area. 491(7423), 212-217. doi:10.1038/nature11527

Leaver, A., Seydell-Greenwald, A., Turesky, T., Morgan, S., Kim, H., \& Rauschecker, J. J. F. i. s. n. (2012). Cortico-limbic morphology separates tinnitus from tinnitus distress. 6, 21. doi:10.3389/fnsys.2012.00021

Leaver, A., Turesky, T., Seydell-Greenwald, A., Morgan, S., Kim, H., \& Rauschecker, J. J. H. b. m. (2016). Intrinsic network activity in tinnitus investigated using functional MRI. 37(8), 2717-2735. doi:10.1002/hbm.23204 
Leaver, A. M., Renier, L., Chevillet, M. A., Morgan, S., Kim, H. J., \& Rauschecker, J. P. (2011). Dysregulation of Limbic and Auditory Networks in Tinnitus. Neuron, 69(1), 33-43. doi:https://doi.org/10.1016/j.neuron.2010.12.002

Leaver, A. M., Seydell-Greenwald, A., \& Rauschecker, J. P. (2016). Auditory-limbic interactions in chronic tinnitus: Challenges for neuroimaging research. Hear Res, 334, 49-57. doi:10.1016/j.heares.2015.08.005

Lefebvre, S., Demeulemeester, M., Leroy, A., Delmaire, C., Lopes, R., Pins, D., . . Jardri, R. (2016). Network dynamics during the different stages of hallucinations in schizophrenia. Hum Brain Mapp, 37(7), 2571-2586. doi:10.1002/hbm.23197

Mahoney, C. J., Rohrer, J. D., Goll, J. C., Fox, N. C., Rossor, M. N., \& Warren, J. D. (2011). Structural neuroanatomy of tinnitus and hyperacusis in semantic dementia. 82(11), 1274-1278. doi:10.1136/jnnp.2010.235473 \%J Journal of Neurology, Neurosurgery \&amp; Psychiatry

Maudoux, A., Lefebvre, P., Cabay, J. E., Demertzi, A., Vanhaudenhuyse, A., Laureys, S., \& Soddu, A. (2012). Auditory resting-state network connectivity in tinnitus: a functional MRI study. PLoS One, 7(5), e36222. doi:10.1371/journal.pone.0036222

Meng, Z., Zheng, Y., Liu, S., Wang, K., Kong, X., Tao, Y., . . . otorhinolaryngology, e. (2012). Reliability and validity of the chinese (mandarin) tinnitus handicap inventory. 5(1), 10-16. doi:10.3342/ceo.2012.5.1.10

Meyer, M., Neff, P., Liem, F., Kleinjung, T., Weidt, S., Langguth, B., \& Schecklmann, M. (2016). Differential tinnitus-related neuroplastic alterations of cortical thickness and surface area. Hear Res, 342, 1-12. doi:10.1016/j.heares.2016.08.016

Mirz, F., Pedersen, B., Ishizu, K., Johannsen, P., Ovesen, T., Stodkilde-Jorgensen, H., \& Gjedde, A. (1999). Positron emission tomography of cortical centers of tinnitus. Hear Res, 134(1-2), 133-144.

Møller, A. J. P. i. b. r. (2007). Tinnitus and pain. 166, 47-53. doi:10.1016/s0079-6123(07)66004-X

Navratilova, E., Xie, J., Okun, A., Qu, C., Eyde, N., Ci, S., . . Porreca, F. J. P. o. t. N. A. o. S. o. t. U. S. o. A. (2012). Pain relief produces negative reinforcement through activation of mesolimbic reward-valuation circuitry. 109(50), 20709-20713. doi:10.1073/pnas.1214605109

Ospina, J., Jalilianhasanpour, R., \& Perez, D. J. H. o. c. n. (2019). The role of the anterior and midcingulate cortex in the neurobiology of functional neurologic disorder. 166, 267-279. doi:10.1016/b978-0-444-64196-0.00014-5

Petacchi, A., Laird, A. R., Fox, P. T., \& Bower, J. M. (2005). Cerebellum and auditory function: an ALE meta-analysis of functional neuroimaging studies. Hum Brain Mapp, 25(1), 118-128. doi:10.1002/hbm.20137

Rauschecker, J., Leaver, A., \& Mühlau, M. J. N. (2010). Tuning out the noise: limbic-auditory interactions in tinnitus. 66(6), 819-826. doi:10.1016/j.neuron.2010.04.032

Rauschecker, J. P., May, E. S., Maudoux, A., \& Ploner, M. (2015). Frontostriatal Gating of Tinnitus and Chronic Pain. Trends Cogn Sci, 19(10), 567-578. doi:10.1016/j.tics.2015.08.002

Schmidt, S., Akrofi, K., Carpenter-Thompson, J., \& Husain, F. J. P. o. (2013). Default mode, dorsal attention and auditory resting state networks exhibit differential functional connectivity in tinnitus and hearing loss. 8(10), e76488. doi:10.1371/journal.pone.0076488

Schmidt, S. A., Akrofi, K., Carpenter-Thompson, J. R., \& Husain, F. T. (2013). Default mode, dorsal attention and auditory resting state networks exhibit differential functional connectivity in tinnitus and hearing loss. PLoS One, 8(10), e76488. doi:10.1371/journal.pone.0076488 
Sommer, I., Diederen, K., Blom, J., Willems, A., Kushan, L., Slotema, K., . . Kahn, R. J. B. a. j. o. n. (2008). Auditory verbal hallucinations predominantly activate the right inferior frontal area. 131, 3169-3177. doi:10.1093/brain/awn251

Song, J., Vanneste, S., \& De Ridder, D. J. P. o. (2015). Dysfunctional noise cancelling of the rostral anterior cingulate cortex in tinnitus patients. 10(4), e0123538. doi:10.1371/journal.pone.0123538

Takahashi, Y., Batchelor, H., Liu, B., Khanna, A., Morales, M., \& Schoenbaum, G. J. N. (2017). Dopamine Neurons Respond to Errors in the Prediction of Sensory Features of Expected Rewards. 95(6), 1395-1405.e1393. doi:10.1016/j.neuron.2017.08.025

Tsai, M., Cai, Y., Wang, C., Zheng, Y., Ou, J., \& Chen, Y. J. B. r. i. (2018). Tinnitus Abnormal Brain Region Detection Based on Dynamic Causal Modeling and Exponential Ranking. 2018, 8656975. doi:10.1155/2018/8656975

Vanneste, S., Alsalman, O., \& De Ridder, D. J. T. J. o. n. t. o. j. o. t. S. f. N. (2019). Top-down and Bottom-up Regulated Auditory Phantom Perception. 39(2), 364-378. doi:10.1523/jneurosci.0966-18.2018

Vogt, B. J. J. o. c. n. (2016). Midcingulate cortex: Structure, connections, homologies, functions and diseases. 74, 28-46. doi:10.1016/j.jchemneu.2016.01.010

Weiler, E. W., Brill, K., Tachiki, K. H., \& Wiegand, R. (2000). Electroencephalography correlates in tinnitus. Int Tinnitus J, 6(1), 21-24.

Yamasaki, H., LaBar, K. S., \& McCarthy, G. (2002). Dissociable prefrontal brain systems for attention and emotion. Proc Natl Acad Sci U S A, 99(17), 11447-11451. doi:10.1073/pnas.182176499

Yang, H., Cai, Y., Guo, H., Xiong, H., Sun, Y., Huang, X., \& Zheng, Y. J. I. j. o. a. (2018). Prevalence and factors associated with tinnitus: data from adult residents in Guangdong province, South of China. 57(12), 892-899. doi:10.1080/14992027.2018.1506169

Yu, E., Liao, Z., Mao, D., Zhang, Q., Ji, G., Li, Y., \& Ding, Z. J. C. A. r. (2017). Directed Functional Connectivity of Posterior Cingulate Cortex and Whole Brain in Alzheimer's Disease and Mild Cognitive Impairment. 14(6), 628-635. doi:10.2174/1567205013666161201201000

Zhou, G. P., Shi, X. Y., Wei, H. L., Qu, L. J., Yu, Y. S., Zhou, Q. Q., . . . Tao, Y. J. (2019). Disrupted Intraregional Brain Activity and Functional Connectivity in Unilateral Acute Tinnitus Patients With Hearing Loss. Front Neurosci, 13, 1010. doi:10.3389/fnins.2019.01010

Zung, W. W. (1971). A rating instrument for anxiety disorders. Psychosomatics, 12(6), 371-379. doi:10.1016/s0033-3182(71)71479-0

Zung, W. W. K. (1986). Zung Self-Rating Depression Scale and Depression Status Inventory. In N. Sartorius \& T. A. Ban (Eds.), Assessment of Depression (pp. 221-231). Berlin, Heidelberg: Springer Berlin Heidelberg. 


\section{Figures}

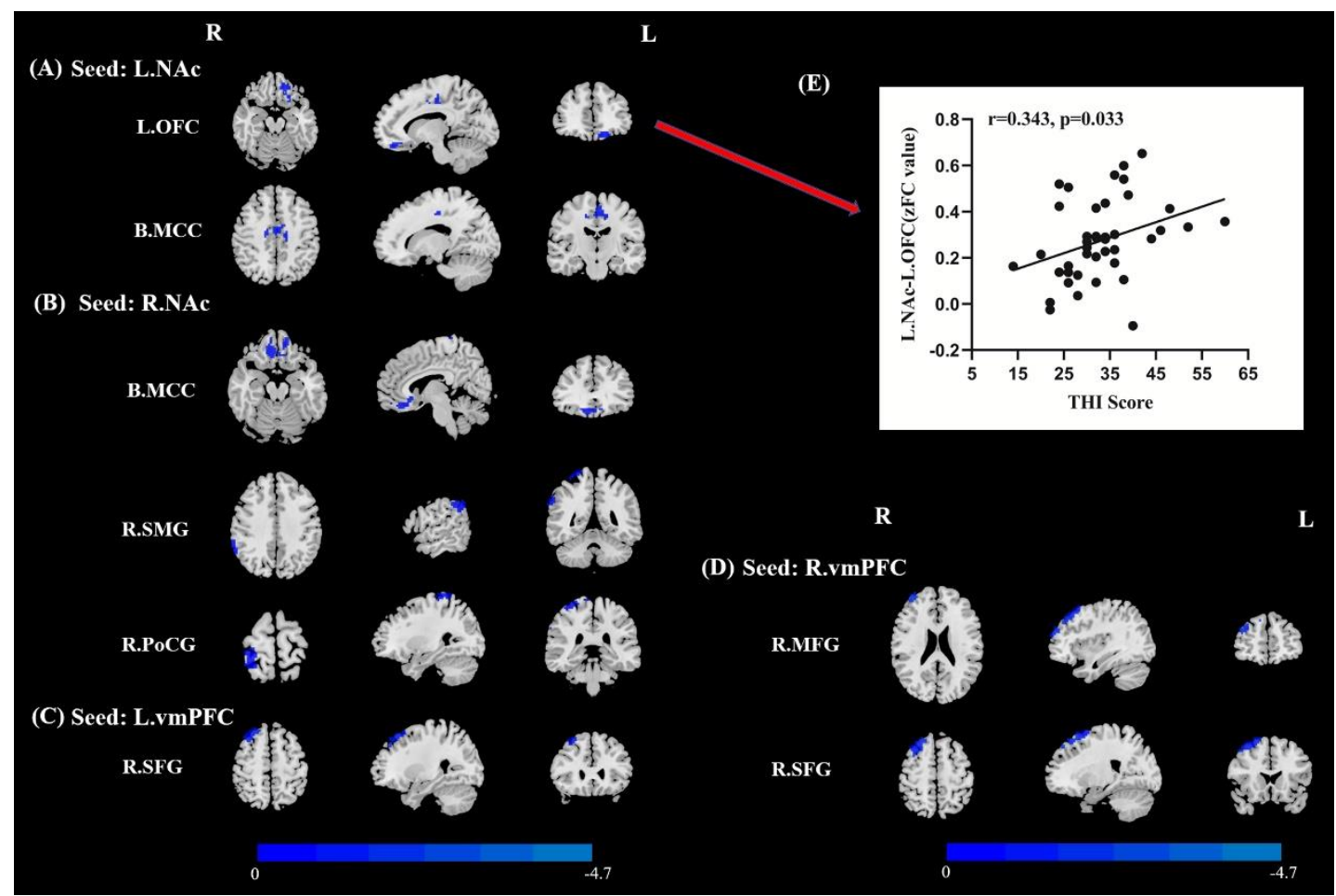




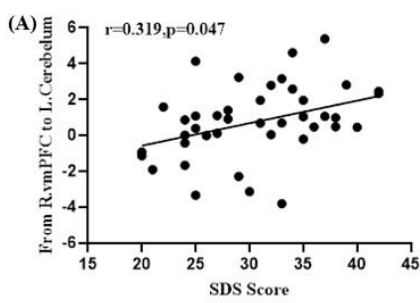

(E)

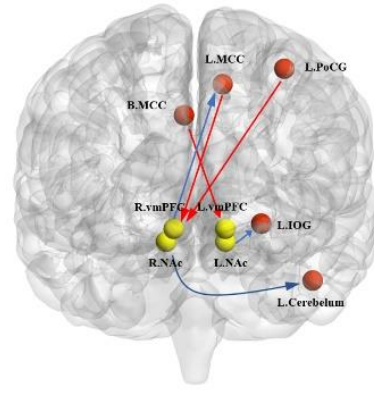

(D)
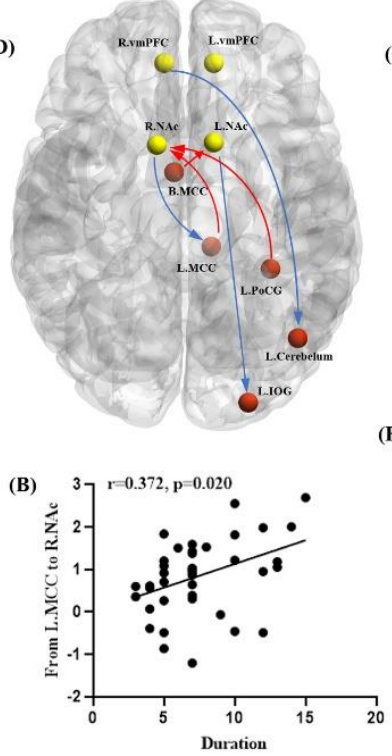

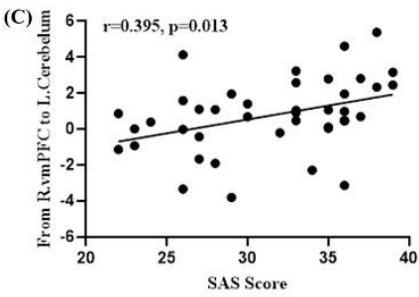

(F)

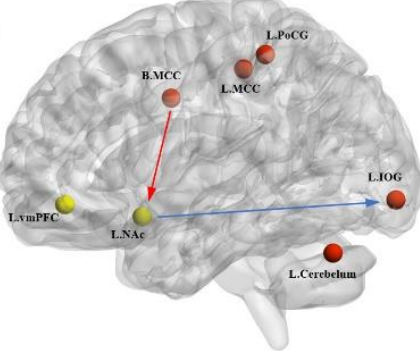




\section{Figures}

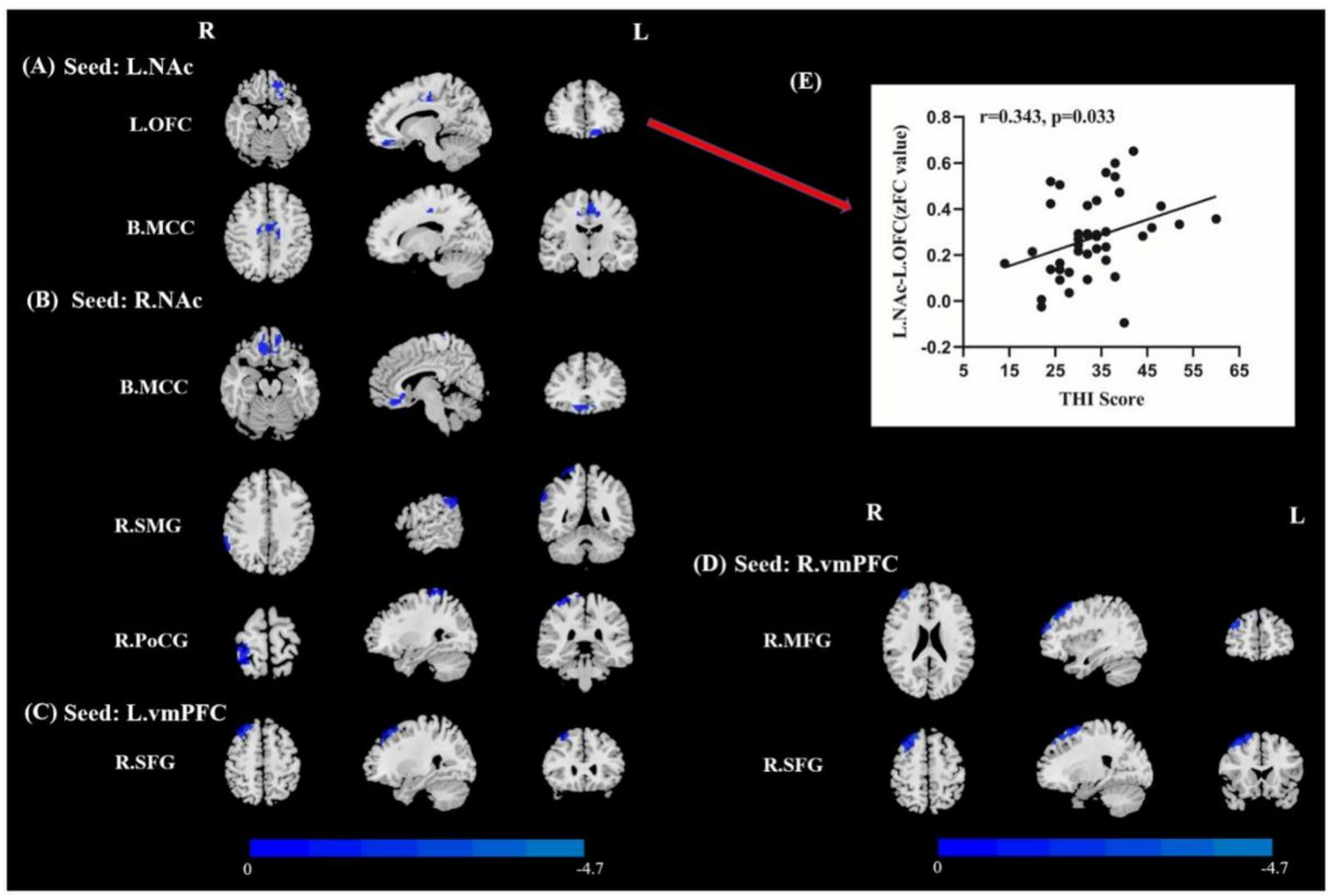

Figure 1

(A-D) The significant FC between the bilateral NAc/vmPFC seed regions and the whole brain (GRF correction, voxel-level $p<0.005$, cluster-level $p<0.05)$. (E) The THI scores were positively correlate with the reduced FC between the left NAc and the left OFC ( $r=0.343, p=0.033)$. L, left; R, right; B. bilateral. Blue means decreased connectivity. The color bar represents the t-value. 
(A)

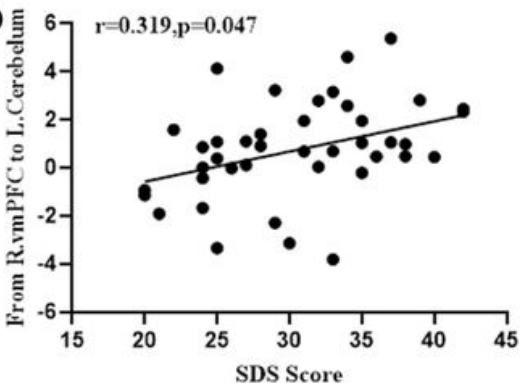

(E)

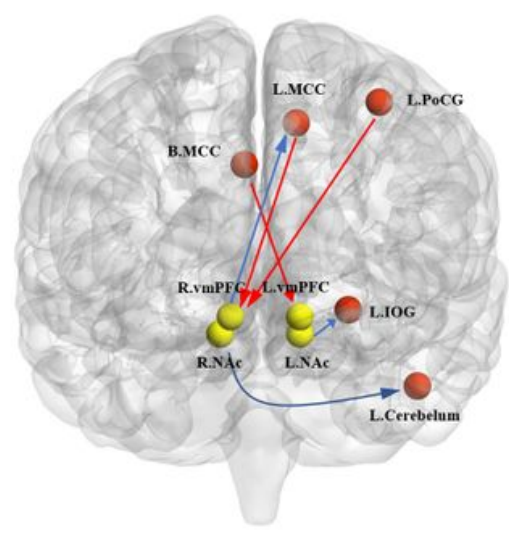

(D)

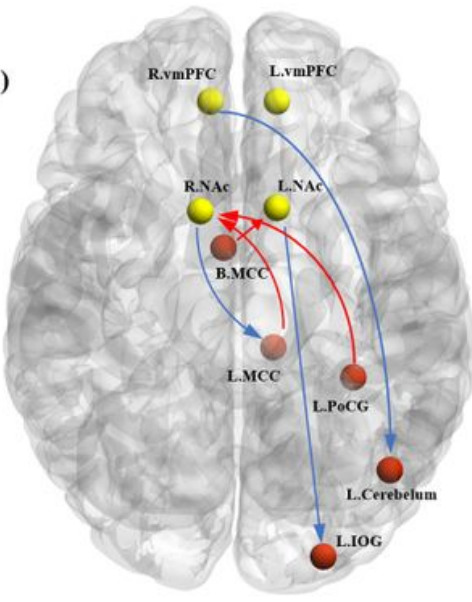

(B)

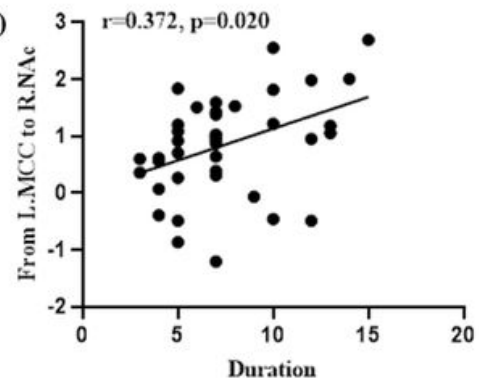

(C)

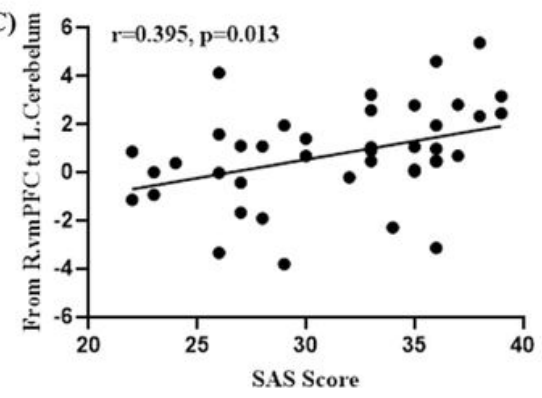

(F)

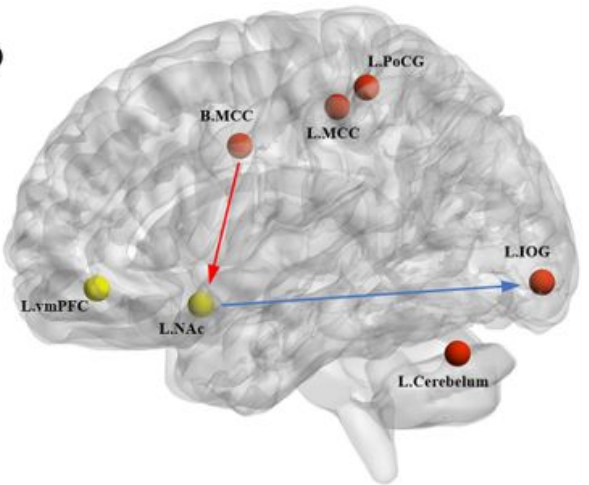

Figure 2

(D-F) The EC of the NAc and vmPFC. The blue line represents the EC from the bilateral NAc/vmPFC to the other brain regions; the red line means the EC from the other brain regions to the bilateral NAc/vmPFC. (A) and (C) The SDS scores and the SAS scores were positively correlate with the decreased effective connectivity from the right vmPFC and the left Cerebelum_Crus1 $(r=0.319, p=0.047 ; r=0.395, p=$ 0.013). (B) Positive correlation between the enhanced effective connectivity from the left MCC to the right NAc $(r=0.372, p=0.020)$.

\section{Supplementary Files}

This is a list of supplementary files associated with this preprint. Click to download.

- TableS1.docx

- Tables2.docx

- TableS3.docx

- Tables4.docx

- TableS5.docx 